EUROPHYSICS LETTERS

Europhys. Lett., (), pp. ()

\title{
Giant Tunneling Magnetoresistance, Glassiness, and the Energy Landscape at Nanoscale Cluster Coexistence
}

\author{
Sanjeev Kumar ${ }^{1}$, Chandra Shekhar Mohapatra ${ }^{2}$ and Pinaki Majumdar ${ }^{3}$ \\ 1 Institute for Physics, Theoretical Physics III, Electronic Correlations and Magnetism, \\ University of Augsburg, 86135 Augsburg, Germany \\ 2 Department of Physics, I.I.T Kharagpur, Kharagpur 721 302, India \\ 3 Harish-Chandra Research Institute, Chhatnag Road, Jhusi, Allahabad 211 019, India \\ (received ; accepted ) \\ PACS. 72.80. $\mathrm{Ng}$ - Disordered solids. \\ PACS. 75.47.Gk - Colossal magnetoresistance. \\ PACS. 75.50.Lk - Spin glasses and other random magnets.
}

\begin{abstract}
. -
We present microscopic results on the giant tunneling magnetoresistance that arises from the nanoscale coexistence of ferromagnetic metallic (FMM) and antiferromagnetic insulating (AFI) clusters in a disordered two dimensional electron system with competing double exchange and superexchange interactions. Our Monte Carlo study allows us to map out the different field regimes in magnetotransport and correlate it with the evolution of spatial structures. At coexistence, the isotropic $O(3)$ model shows signs of slow relaxation, and has a high density of low energy metastable states, but no genuine glassiness. However, in the presence of weak magnetic anisotropy, and below a field dependent irreversibility temperature $T_{i r r}$, the response on field cooling (FC) differs distinctly from that on zero field cooling (ZFC). We map out the phase diagram of this 'phase coexistence glass', highlight how its response differs from that of a standard spin glass, and compare our results with data on the manganites.
\end{abstract}

Introduction. - First order phase transitions involve a regime of metastability and phase coexistence. Two phases, either both ordered, or one ordered and the other disordered, continue to be minima of the free energy and the system can get trapped in the metastable minimum, or be in a state of macroscopic coexistence of the two phases. This scenario is complicated by the presence of disorder. Disorder introduces preferential pinning or 'nucleation' centers for the two phases, and require us to consider the energy $(\mathcal{E})$ as a functional of the full order parameter field, $\phi_{\mathbf{r}}$, say $[1,2,3]$. The result is a pattern of coexisting clusters in real space, and a rugged landscape for $\mathcal{E}\left\{\phi_{\mathbf{r}}\right\}$ with many deep local minima in configuration space. The non trivial energy landscape results in slow relaxation and history dependence in the response of the system, the generic signatures of 'glassiness'. Such connection between phase coexistence and glassy effects has been explored in detail in vortex matter in superconductors [4], and more recently in the 'colossal magnetoresistance' manganites $[5,6,7]$.

Typeset using EURO-TEX 
Electron systems at coexistence, e.g, the manganites, involve charge degrees of freedom, and, in addition to glassiness, raise the possibility of a dramatic electrical response and insulator-metal transitions. It has been established that there is coexistence of ferromagnetic metallic (FMM) and charge ordered antiferromagnetic insulating (CO-AF-I) clusters in the low $T_{c}$ manganites $[8,9]$, notably the $\mathrm{La}_{1-x-y} \mathrm{Pr}_{y} \mathrm{Ca}_{x} \mathrm{MnO}_{3}$ family. These materials exhibit 'out of equilibrium' features like irreversibility [5], slow relaxation [6], and persistent field memory [7]. In addition, since conduction depends on electron tunneling between "half-metallic" FMM clusters, a weak magnetic field greatly enhances the conductance [8] by aligning the cluster moments. The field induced suppression of resistivity $(\rho)$ can attain $\rho(0) / \rho(h) \sim 10^{2}$, at fields $h \sim 1$ Tesla, down to zero temperature. Coexistence, glassiness and giant low temperature tunneling magnetoresistance (TMR) are intimately related.

A first principles theory capturing nanoscale coexistence, TMR, and the memory effects requires a method which can handle multiple interactions, quenched disorder, and thermal effects simultaneously. In this paper we use a recently developed Monte Carlo (MC) technique [10] to study a model of competing double exchange (DE) and superexchange (SE) in the background of weak disorder, and clarify the following: $(i)$ the origin and magnitude of the large TMR at cluster coexistence and the high field evolution of the magnetic state, and $(i i)$ the nature of the "phase coexistence glass", that arises from a non trivial energy landscape and magnetic anisotropy, and its difference with respect to a canonical spin glass.

Earlier work. - Models of competing DE and SE, in the presence of weak disorder, have been studied in the recent past via 'exact' simulation on small lattices [11]. These studies suggest spatial coexistence of pinned clusters of the competing ordered phases. However, being limited to very small sizes, they are unable to clarify the cluster distribution, the transport properties, or possible memory effects in the system.

In the absence of a microscopic approach, a resistor network phenomenology [11] has been developed to model transport at coexistence. Although useful as a starting approximation, it is unable to take into account the 'spin overlap' between clusters that controls magnetotransport, or the spatial correlations in the cluster distribution. We also know of no microscopic calculation addressing the slow relaxation and glassiness in the coexistence regime. In this paper we extend our earlier study at zero field [12] to clarify the magnetotransport and explore the history dependence in the coexistence regime.

Model and method. - We address the issues of TMR and 'glassiness' using the following model [12] of competing DE and SE in the presence of weak disorder in two dimension (2D):

$$
H=-t \sum_{\langle i j\rangle, \sigma} c_{i \sigma}^{\dagger} c_{j \sigma}+\sum_{i}\left(\epsilon_{i}-\mu\right) n_{i}-J_{H} \sum_{i} \mathbf{S}_{i} \cdot \vec{\sigma}_{i}+J_{S} \sum_{\langle i j\rangle} \mathbf{S}_{i} \cdot \mathbf{S}_{j}-h \sum_{i} S_{i z}+H_{a n i s}
$$

The hopping is $t=1$ for nearest neighbours, and sets our basic energy scale, and the structural disorder $\epsilon_{i}$ is uniformly distributed between $\pm \Delta / 2$. $J_{H}$ is the Hunds coupling, $J_{S}$ is the AF superexchange, and $h$ is the applied magnetic field. We will use $H_{a n i s}=-\alpha \sum_{i} S_{i z}^{2}$, when studying the effect of anisotropy.

We assume $J_{H} / t \rightarrow \infty$ and a classical core spin with $\left|\mathbf{S}_{i}\right|=1$. It is known that using finite $J_{H} / t$ [13], or a 'quantum' $S=3 / 2$ core spin [13] does not affect the principal observations regarding FMM-AFI phase competetion. We also ignore the field coupling to electron spin since that only renormalises the net moment. Projecting out fermion states at $+J_{H} / 2$ we obtain $H_{e l}=-t \sum_{\langle i j\rangle} f_{i j}\left(e^{i \Phi_{i j}} \gamma_{i}^{\dagger} \gamma_{j}+h . c\right)+\sum_{i}\left(\epsilon_{i}-\mu\right) n_{i}$. The $\gamma$ are spinless fermions, and the hopping amplitude, $g_{i j}=f_{i j} e^{i \Phi_{i j}}$, between locally aligned states, can be written in terms of the polar angle $\left(\theta_{i}\right)$ and azimuthal angle $\left(\phi_{i}\right)$ of the $\operatorname{spin} \mathbf{S}_{i}$ as $\cos \frac{\theta_{i}}{2} \cos \frac{\theta_{j}}{2}+\sin \frac{\theta_{i}}{2} \sin \frac{\theta_{j}}{2} e^{-i\left(\phi_{i}-\phi_{j}\right)}$. 

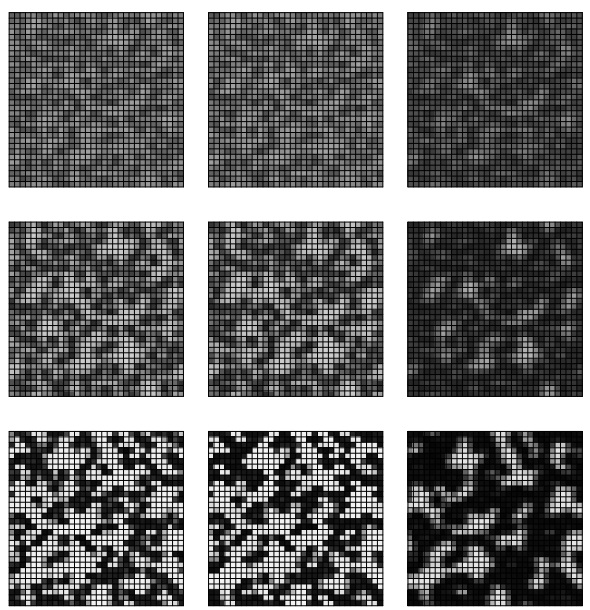

Fig. 1. - Cluster pattern for $n=0.1$ in the isotropic model. We show the spatially resolved nearest neighbour spin correlation $f\left(\mathbf{R}_{i}\right)=\left\langle\mathbf{S}\left(\mathbf{R}_{i}\right) \cdot \mathbf{S}\left(\mathbf{R}_{i}+\hat{x}\right)\right\rangle$. The system size is $32 \times 32$. Field variation along the row: $h=0.0,0.01,0.10$, left to right, and, down the column, the temperature are $T=$ 0.06, 0.03, 0.01. Dark regions are FMM, white are AF, grey are paramagnetic.

The magnitude of the hopping is $f_{i j}=\sqrt{\left(1+\mathbf{S}_{i} \cdot \mathbf{S}_{j}\right) / 2}$, while the phase is specified by $\tan \Phi_{i j}=\operatorname{Im}\left(g_{i j}\right) / \operatorname{Re}\left(g_{i j}\right)$. We use the same technique as in [12] to construct an explicit classical spin Hamiltonian, with self consistently computed couplings, from the spin-fermion problem: $H_{e f f}=-\sum_{\langle i j\rangle} D_{i j} f_{i j}+J_{S} \sum_{\langle i j\rangle} \mathbf{S}_{i} . \mathbf{S}_{j}-h \sum_{i} S_{i z}$. The exchange $D_{i j}$ are determined self consistently as the thermal average of $\hat{\Gamma}_{i j}=\left(e^{i \Phi_{i j}} \gamma_{i}^{\dagger} \gamma_{j}+h . c\right)$ over configurations generated by $H_{e f f}$. The $D_{i j}$ 's that emerge at consistency depend on temperature $(T)$ and the magnetic field, and are strongly inhomogeneous and spatially correlated. Our transport calculation is based on an exact implementation of the Kubo formula described in detail elsewhere [14].

At weak $J_{S}$, the competing phases in the clean problem are a FMM for $n \geq n_{c}$ (a critical density), and an AFI at $n=0$. There is a discontinuous jump in $n$ from $n=0$ to $n=n_{c}$ across the AFI-FMM transition. Disorder broadens the transition, leading to a regime of FMM-AFI cluster coexistence. In this paper, as earlier [12], we set $J_{S}=0.05$ and $\Delta=1.0$, choose $n=0.1$ to be at the center of the coexistence regime, and focus on the effects of finite $h$.

Results. - Our results are mainly in two parts: (A). the effect of $h$ on transport and magnetisation in the fully self-consistent isotropic model, and (B). results in the presence of magnetic anisotropy, employing the spatially correlated bonds obtained in (A).

(A). Fully self-consistent calculation without anisotropy: Fig.1 shows the spatially resolved nearest neighbour spin correlation $f_{s}\left(\mathbf{R}_{i}\right)=\left\langle\mathbf{S}\left(\mathbf{R}_{i}\right) . \mathbf{S}\left(\mathbf{R}_{i}+\hat{x}\right)\right\rangle$. The dark regions have strong FM correlations, the white regions are $\mathrm{AF}$, while the grey regions are paramagnetic. The pattern is shown for three combinations of $h$ and $T$. We interpret the spatial patterns as follows.

(i) In the high $T$ paramagnetic phase (top row) $f_{s}$ is small on all links since $T$ is much larger than the exchange scales, and it is only at $h=0.10$ (extreme right) that we see signs of magnetisation. The response is $T$ or $h$ dominated, with the magnetic correlations not playing any essential role. (ii) The "contrast" between FMM and AFI regions improves with cooling 

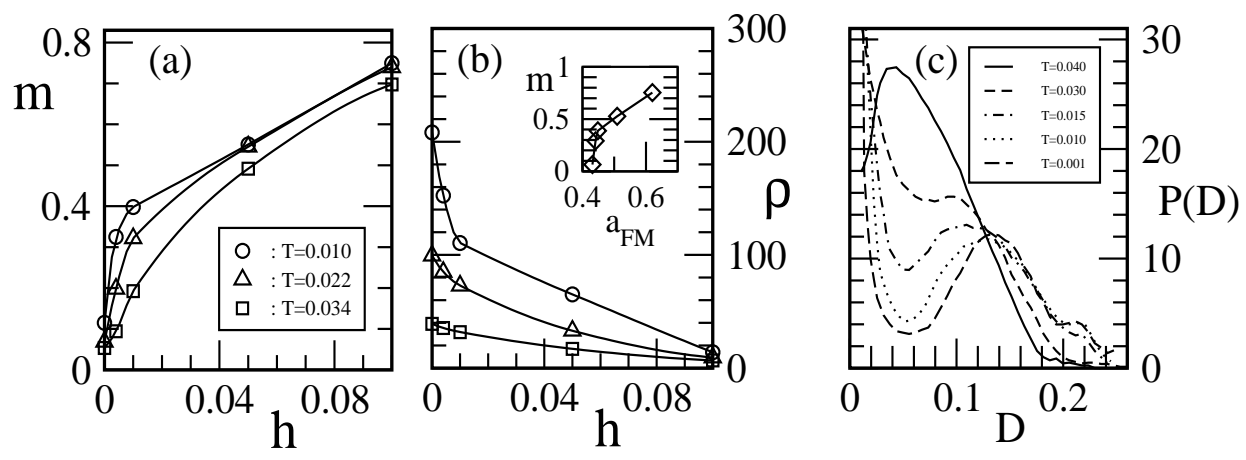

Fig. 2. - Magnetism and transport in the isotropic model: $n=0.1$ and $\Delta=1.0$. (a) $h$ dependence of magnetisation $m(h, T)$ after ZFC, $(b) h$ dependence of resistivity $\rho(h, T)$ after ZFC. Note the sharp low field response. The inset to $(b)$ shows the growth in $m(h)$ correlated with the change in FMM 'surface coverage' at $T=0.01$. Consistent with Fig.1, the FMM regions hardly grow at low $h$, while $m(h)$ increases rapidly. $(c)$ The bond distribution $P(D, T)$ obtained from the self-consistent calculation.

(central row) and the segregation into FMM and AFI clusters becomes apparent. Low field, $h \sim 0.01$ (centre), does not have any perceptible effect on $f_{s}$ compared to $h=0$ (left), but $h=0.10$ (right) leads to a large magnetisation. (iii) At the lowest $T$ (bottom row), the $h=0$ cluster pattern is very distinct with the electrons confined to the FMM clusters. A low field does not lead to a significant difference in the cluster pattern but a large change in $m(h)$ as we discuss next. Large field, $h=0.10$, leads to a visible increase in the FMM volume fraction.

The insensitivity of the cluster pattern to weak fields, at all $T$, should be seen in conjunction with the response in magnetism, $m(h, T)$, and transport, $\rho(h, T)$, in Fig.2.(a)-(b). Within each FMM cluster the moments are aligned at low $T$, but the overall $m(T)$ is small at $h=0$ due to the random orientation of the "cluster moments". The effect of a weak field, applied after cooling the system, is to orient the cluster moments parallel to the field. Since the clusters typically have $>10$ spins coupled together even a weak field leads to a sharp increase in the magnetisation. In addition, the highly resistive state at $h=0$, which inhibited electron tunneling due to random orientation of the cluster moments, now rapidly turns conducting due to increasing spin overlap between clusters. The rotation of cluster moments, and the resulting TMR, is the predominant low temperature zero field cooled (ZFC) effect in this system.

When a larger field is applied after cooling the system two other effects come into play. The FMM phase grows in volume, and residual spin disorder (due to finite $T$ ) within the clusters gets suppressed. The system now behaves almost like a canonical 'clean' DE ferromagnet, $m(h, T)$ nears saturation and $\rho(h, T)$ drops to a low value controlled by the structural disorder.

Just as the spin correlations in Fig.1 evolve with changing $T$, the bonds $D_{i j}$ also depend on temperature (through the self-consistency condition). Fig.2.(c) shows the evolution of the system averaged bond distribution $P(D)$ with changing $T$. Note that $P(D)$, although non trivial, in itself has no information about the spatial correlation between bonds.

Using MC runs with equilibriation time $\tau_{e q} \sim 10^{3}$ steps revealed an apparent difference between $\mathrm{ZFC}$ and $\mathrm{FC}$ in this system. However, with increasing $\tau_{e q}$ this difference diminishes, and for $\tau_{e q} \geq 10^{4}$ the $\mathrm{FC}$ and ZFC response match. It is known in the context of spin glasses that there cannot be any metastability in a $O(3)$ model in 2D [15]. Nevertheless, the long 'relaxation time' (in the MC sense) suggests that the effective magnetic model probably has 

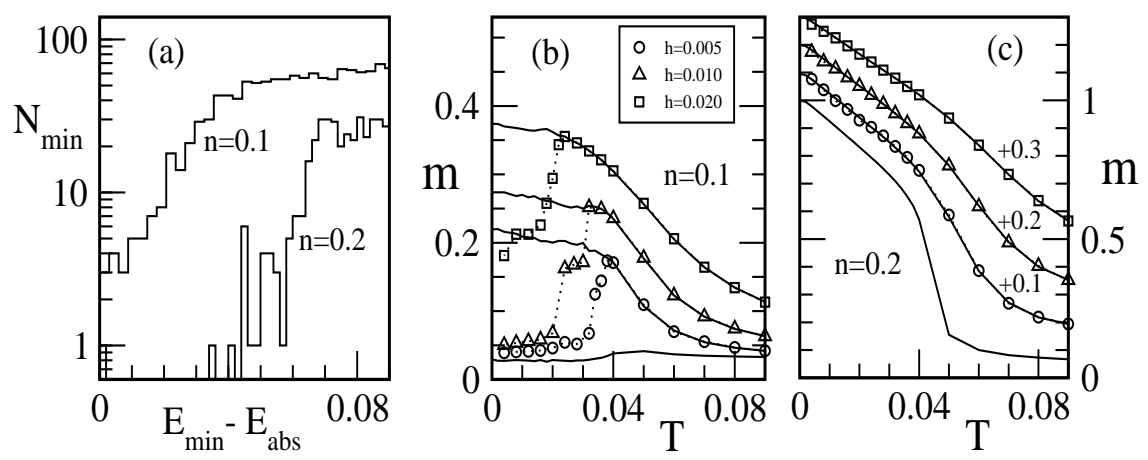

Fig. 3. - Metastable states, and memory effects in the anisotropic model: (a) The density, $N_{\text {min }}$, of metastable low energy minima at $n=0.1$ (cluster coexistence) and $n=0.2$ (almost homogeneous FMM) obtained in the isotropic model. The energy $E_{m i n}-E_{a b s}$ is measured with respect to the 'absolute' minimum in the respective case. The results are qualitatively similar in the anisotropic model. (b) Magnetic response in the weakly anisotropic model, at $n=0.1$, using bonds obtained from the self-consistent calculation. Firm lines are for FC, dotted lines for ZFC. (c) FC-ZFC response in the $n=0.2$ (nearly homogeneous FMM) case, including anisotropy. In this case the FC and ZFC response are identical, consistent with the absence of low energy metastable states (panel $(a)$ ).

a non trivial energy landscape. To gain a deeper understanding of the observed relaxation we examined the density of low energy metastable configurations (LEMC), Fig.3.(a). We used the low $T, h=0$, energy functional $\mathcal{E}\{\theta, \phi\}=-\sum_{\langle i j\rangle} D_{i j} f_{i j}+J_{S} \sum_{\langle i j\rangle} \mathbf{S}_{i} . \mathbf{S}_{j}$, and employed a conjugate gradient method to locate local minima starting from random initial configurations. Since this is an expensive effort for continuous spin systems, we restrict our results to system size $8 \times 8$, using upto $10^{4}$ initial configurations. For the system at coexistence, $n=0.1$, the density of LEMC is very high. We also studied the LEMC in our model with electron density $n=0.2$ (an almost homogeneous FMM) and found that there is a clear gap between the absolute minimum and the first metastable state. The high density of LEMC at $n=0.1$ suggests that even weak anisotropy might stabilise glassiness, which we discuss next.

(B). Anisotropic model with correlated bonds: The full self consistent calculation, involving iterative bond evaluation, is difficult to perform in systems where $\tau_{e q}$ is large. However, the qualitative character of the bond distribution, Fig.2.(c), computed self-consistently, does not change for $T<0.02$. Therefore, to clarify the interplay of "clustering" (i.e, spatial correlation between the bonds) and magnetic anisotropy we studied the following model via classical MC:

$$
H_{\text {corr }}=-\sum_{i j} D_{i j}^{\text {corr }} f_{i j}+J_{S} \sum_{i j} \mathbf{S}_{i} . \mathbf{S}_{j}-h \sum_{i} S_{i z}+H_{a n i s} .
$$

The bonds $D_{i j}^{\text {corr }}$ are obtained from the self consistent scheme at low $T$. They are spatially correlated, with the kind of spatial clustering seen in Fig.1. This model ignores the thermal and field evolution of the $D_{i j}$ that is seen in the fully consistent calculation.

We studied the FC-ZFC difference in this model, Fig.3.(b), with bonds corresponding to $n=0.1$. Even with $\tau_{e q}>10^{4}$ and averaging time $\tau_{a v}>10^{4}$, the ZFC response differs clearly from the FC response. Below a temperature $T_{i r r}(h)$, the $\mathrm{FC}$ and $\mathrm{ZFC}$ results are distinct, with $m(T, h)$ being higher in the FC case. The relative difference between FC and ZFC on $m(T, h)$, at any $T$, decreases with increasing $h$, while the 'irreversibility temperature', $T_{i r r}(h)$, reduces with increasing $h$, vanishing at $h \sim 0.04$. For the $n=0.2$ case the FC and ZFC results 

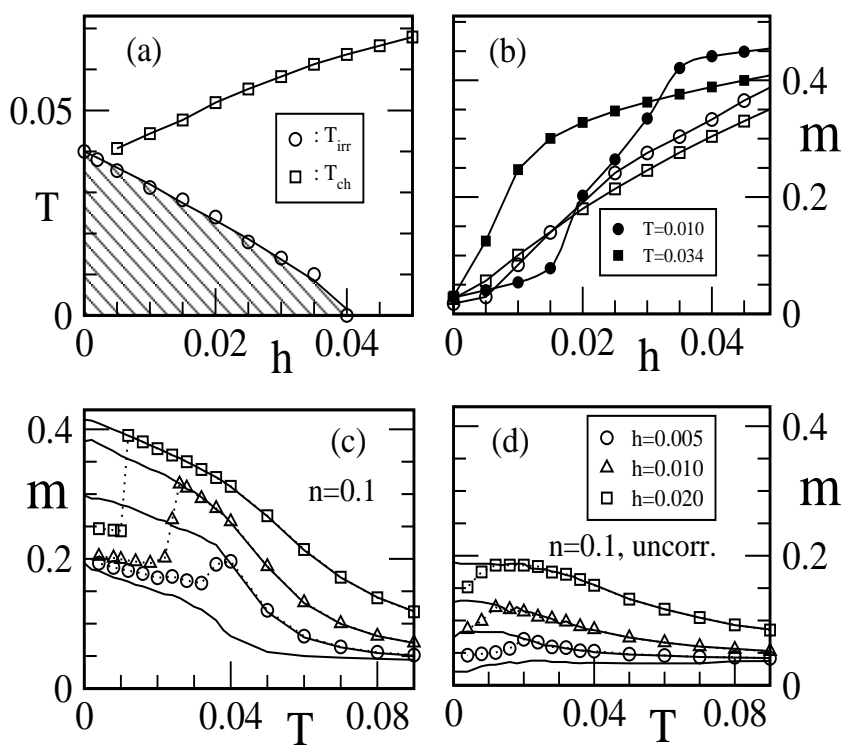

Fig. 4. $-(a)$ The $h-T$ phase diagram with anisotropy, $\alpha=0.02$. The shaded area is the regime of irreversibility, bounded by the irreversibility temperature $T_{i r r}(h)$. The upper curve refers to the 'upturn' in the FM response. (b) Field response after ZFC computed with correlated bonds (filled symbols) and uncorrelated bonds (open symbols). $(c)-(d)$ Comparing FC-ZFC response between the PCG and homogeneous SG, $(c)$ response obtained with correlated bonds for the $n=0.1$ case, and $(d)$ the model with uncorrelated bonds $D_{i j}^{S G}$ picked from $P(D)$. All these systems have size $24 \times 24$, $J_{S}=0.05$, and uniaxial anisotropy with $\alpha=0.02$.

are indistinguishable, Fig.3.(c), consistent with the simple energy landscape.

Fig.4.(a) puts together the $h-T$ phase diagram that describes our phase coexistence glass (PCG) in the presence of anisotropy, with the shaded region denoting the regime of irreversibility. The difference between FC and ZFC is well known in spin glasses [15], and has also been observed [5] in the phase separated manganites. The key question, however, is whether the glassiness observed at coexistence can be understood in terms of the standard categories of $(a)$ a homogeneous spin glass (SG), or $(b)$ a 'superparamagnet' (SP), or $(c)$ a cluster glass (CG). Let us analyse our spatial patterns and magnetic results with reference to these models, as well as experimental data on the manganites.

(a) Since we do not compute time dependent responses to characterise our glassy state we cannot compare directly with relaxational results known for spin glasses, etc. We instead studied a specific 'spin glass' model, derived from the coexistence problem: $H_{S G}=$ $-\sum_{i j} D_{i j}^{S G} f_{i j}+J_{S} \sum_{i j} \mathbf{S}_{i} . \mathbf{S}_{j}-h \sum_{i} S_{i z}+H_{a n i s}$. In contrast to the PCG, the $D_{i j}^{S G}$ are picked at random from $P(D)$ without retaining any spatial correlations. There are three features that distinguish this 'SG' from the PCG: $(i)$ by construction there is no spatial clustering in this model and patterns as in Fig.1 do not emerge, $(i i)$ the response in $m(h)$ after ZFC is much sharper in the PCG compared to the SG, Fig.4.(b), and (iii) the overall magnitude of $m(T, h)$ and the FC-ZFC difference are significantly larger in the PCG compared to the SG, Fig.4.(c)-(d). These differences have their origin in the 'clustering' that characterises the PCG so let us contrast our results with standard features of inhomogeneous systems, a SP or a CG.

(b) The usual superparamagnet is a collection of magnetic nanoparticles, with strong fer- 
romagnetic coupling within each particle (or cluster), while the coupling between clusters is negligible. The low $T$ weak field response of our PCG (in terms of domain rotation) has some similarity with a SP, but at intermediate field or temperature, see Fig.1, the correlation between 'clusters' is important.

(c) A cluster glass extends the SP picture to incorporate intercluster interaction. The detailed physical effects in a CG depend on the density of clusters and their size distribution [16] but the basic identity of the clusters, and the coupling between them, do not depend on $T$ or $h$. The phenomenology of a cluster glass, in terms of spatial correlation and sharp low field response, is closest to our results. The differences are: $(i)$ the entities in the PCG are elementary spins, albeit 'clustered', but not predefined composite objects, and (ii) the "cluster distribution" in the PCG, as well as the coupling between clusters, depends on temperature and field, effectively tuning the DE-SE competetion. That does not happen in a CG.

Our model captures some of the key aspects of field response and glassiness at coexistence in the manganites: the field regimes observed in $\mathrm{Pr}_{0.7} \mathrm{Ca}_{0.3} \mathrm{MnO}_{3}$ [5] including low field anisotropy, followed by domain rotation and then growth of the FM regions, are all seen in our Fig.2-3. We can also capture the behaviour of $T_{i r r}(h)$. We do not have data on the time dependence of magnetic relaxation, as measured in [6], but our results provide insight on how the DE-SE interaction evolves in a clustered system with variation in temperature. Finally, in contrast to bulk measurements, which can only hypothesise about spatial structures, we can correlate the bulk properties with the underlying microscopic situation.

To conclude, we have studied a model of competing double exchange and superexchange in a weakly disordered 2D electron system and characterised the glassy features of the resulting magnetic state. Our results describe how magnetic anisotropy and the non trivial energy landscape at phase coexistence combine to generate several of the glassy features observed in the manganites. It would be interesting to study the detailed time dependent response of this model in $3 \mathrm{D}$ to clarify the outstanding memory effects $[6,7]$ observed in the manganites.

We acknowledge use of the Beowulf cluster at H.R.I. SK gratefully acknowledges support by the Deutsche Forschungsgemeinschaft through SFB484.

\section{REFERENCES}

[1] Y. Imry and S.-k. Ma, Phys. Rev. Lett. 35, 1399 (1975).

[2] Y. Imry and M. Wortis, Phys. Rev. B 19, 3580 (1979).

[3] E. Dagotto, Nanoscale Phase Separation and Colossal Magnetoresistance, Springer (2003).

[4] A. Soibel, et al., Phys. Rev. Lett. 87, 167001 (2001).

[5] I. G. Deac, et al., Phys. Rev. B 63, 172408 (2001).

[6] F. Rivadulla, et al., Phys. Rev. Lett. 93, 167206 (2004).

[7] P. Levy, et al., Phys. Rev. Lett. 89, 137001 (2002).

[8] M. Uehara, et al., Nature, 399, 560 (1999).

[9] M. Fath, et al., Science, 285, 1540 (1999).

[10] S. Kumar and P. Majumdar, cond-mat 0305345.

[11] M. Mayr, et al., Phys. Rev. Lett. 86, 135 (2000).

[12] S. Kumar and P. Majumdar, Phys. Rev. Lett. 92, 126602 (2004).

[13] E. Dagotto, et al., Phys. Rev. B 58, 6414 (1998).

[14] S. Kumar and P. Majumdar, cond-mat 0504656.

[15] J. A. Mydosh, Spin Glasses: An Experimental Introduction, Taylor \& Francis, London (1993).

[16] M. Ulrich, et al., Phys. Rev. B 67, 024416 (2003). 